\title{
The star formation histories of red and blue low surface brightness disk galaxies
}

\author{
G. H. Zhong ${ }^{1,2,3}$, Y. C. Liang ${ }^{1,2}$, F. Hammer ${ }^{4}$, X. Y. Chen ${ }^{1,2,3}$, L. C. Deng ${ }^{1,2}$, and H. Flores ${ }^{4}$ \\ 1 National Astronomical Observatories, Chinese Academy of Sciences, A20 Datun Road, 100012 Beijing, PR China \\ e-mail: [ghzhong;ycliang@nao.cas.cn] \\ 2 Key Laboratory of Optical Astronomy, National Astronomical Observatories, Chinese Academy of Sciences, 100012 Beijing, \\ PR China \\ 3 Graduate School of the Chinese Academy of Sciences, 100049 Beijing, PR China \\ 4 GEPI, Observatoire de Paris-Meudon, 92195 Meudon, France
}

Received 4 February 2010 / Accepted 23 April 2010

\section{ABSTRACT}

\begin{abstract}
Aims. We study the star formation histories (SFH) and stellar populations of 213 red and 226 blue nearly face-on low surface brightness disk galaxies (LSBGs), which are selected from the main galaxy sample of Sloan Digital Sky Survey (SDSS) Data Release Seven (DR7). We also compare the stellar populations and SFH between the two groups.

Methods. The sample of both red and blue LSBGs have sufficient signal-to-noise ratio in the spectral continua. We obtain their absorption-line indices (e.g. $\left.\mathrm{Mg}_{2}, \mathrm{H} \delta_{A}\right), D_{n}(4000)$ and stellar masses from the MPA/JHU catalogs to study their stellar populations and SFH. Moreover we fit their optical spectra (stellar absorption lines and continua) by using the spectral synthesis code STARLIGHT on the basis of the templates of simple stellar populations (SSPs).

Results. We find that red LSBGs tend to be relatively older, of higher metallicity, are more massive and have a higher surface mass density than blue LSBGs. The $D_{n}(4000)-\mathrm{H} \delta_{A}$ plane shows that perhaps red and blue LSBGs have different SFH: blue LSBGs are more likely to experience a sporadic star formation event now, whereas red LSBGs are more likely to have formed stars continuously over the past 1-2 Gyr. Moreover, the fraction of galaxies that experienced recent sporadic formation events decreases with increasing stellar mass. Furthermore, two sub-samples are defined for both red and blue LSBGs: the sub-sample within the same stellar mass range of $9.5 \leq \log \left(M_{\star} / M_{\odot}\right) \leq 10.3$, and the surface brightness limiting sub-sample with $\mu_{0}(R) \geq 20.7 \mathrm{mag} \operatorname{arcsec}^{-2}$. They show consistent results with the total sample in the corresponding relationships, which confirm that our results of the comparasion of the blue and red LSBGs are robust.
\end{abstract}

Key words. galaxies: evolution - galaxies: spiral - galaxies: starburst - galaxies: star formation - galaxies: stellar content

\section{Introduction}

Galaxies with a central surface brightness much fainter than $\mu_{0}(B)=21.65 \pm 0.3 \mathrm{mag} \operatorname{arcsec}^{-2}$ are well known as low surface brightness galaxies (LSBGs). Although they are faint compared to the night sky and hard to find, they represent a significant fraction of the number density of galaxies in the universe (O'Neil \& Bothun 2000; Trachternach et al. 2006) and may comprise up to half of the local galaxy population (McGaugh et al. 1995).

The most widely studied LSBGs are blue (e.g. Zackrisson et al. 2005; Vorobyov et al. 2009), which means that they appear to have a lower metallicity (Burkholder et al. 2001; Haberzettl et al. 2007), lower star formation rate (O'Neil et al. 2007), are evolving much more slowly (van den Hoek et al. 2000), have larger gas fraction (McGaugh \& de Blok 1997; Schombert et al. 2001), lower galaxy density (Rosenbaum et al. 2009) and larger amounts of dark matter (de Blok \& McGaugh 1997) than what is typically found in normal galaxies.

The wide-field CCD survey of O'Neil et al. (1997) firstly found several red LSBGs with optical colors compatible with those seen in old stellar populations. Below we list some works which compared the properties of the two groups. Based on the optical-near infrared color-color diagrams of two red and three blue LSBGs, Bell et al. (1999) found that red and blue LSBGs have different star formation histories (SFH): blue LSBGs are well described by models with a low, roughly constant star formation rate, whereas red LSBGs are better described by a "faded disk" scenario. Furthermore, with 5 red LSBGs, Bell et al. (2000) suggested that the red LSBGs cataloged by O'Neil et al. (1997) are a heterogeneous group, which seem to have relatively few common traits.

Due to the considerable uncertainty regarding the SFH of LSBGs, further studies on the properties of red and blue LSBGs, especially their spectroscopic properties, are important for understanding their formation and evolution. Moreover, the comparison of their SFH presents a good opportunity for understanding the global properties of low surface brightness systems.

Nevertheless, the previous studies of red and blue LSBGs have been traditionally carried out with very small samples. With the advent of the large sky survey of the Sloan Digital Sky Survey (SDSS), it is now possible to dramatically extend these studies in size. Moreover, this enormous amount of highquality data will be undoubtedly important to allow us to study the photometric and spectroscopic properties of those galaxies more carefully. We have selected a large sample of LSBGs (Zhong et al. 2008) from SDSS-DR4 (Adelman-McCarthy et al. 2006), which consists of much more red and blue LSBGs than before. In this work, we select red and blue LSBGs from the 
latest data release of SDSS, the Data Release Seven (DR7) ${ }^{1}$ (Abazajian et al. 2009), which greatly extends the sample. This large sample of galaxies will be helpful to explore and compares the SFH of red and blue LSBGs through their photometric properties and spectral features, such as the relations of $\mathrm{Mg}_{2}$ vs. $\log M_{*}, D_{n}(4000)$ vs. $\mathrm{H} \delta_{A}, D_{n}(4000)$ vs. $\log M_{*}$, surface density and spectral synthesis etc.

This paper is organized as follows. In Sect. 2, we describe the selection of the sample. In Sect. 3 we study the SFH of red and blue LSBGs from some property parameters, including stellar absorption indices, $D_{n}(4000)$, stellar mass, and surface mass density. The stellar populations, studied using the spectral synthesis of the STARLIGHT ${ }^{2}$ code and the simple stellar populations (SSPs) of Bruzual \& Charlot (2003), are given in Sect. 4. The properties of two sub-samples (surface brightness limiting $\left(\mu_{0}(R)\right)$ and stellar mass limiting) are given in Sect. 5. Then we discuss our results in Sect. 6 and summarize this work in Sect. 7.

Throughout the paper, a cosmological model with $H_{0}=$ $70 \mathrm{~km} \mathrm{~s}^{-1} \mathrm{Mpc}^{-1}, \Omega_{\mathrm{M}}=0.3$ and $\Omega_{\lambda}=0.7$ is adopted. All the magnitudes, given in Petrosian magnitudes, and the colors presented here are corrected for Galactic extinction and $K$-correction by using the reddening maps of Schlegel et al. (1998) and the code provided by Blanton et al. (2003), respectively.

\section{The sample}

The SDSS is the most ambitious astronomical survey ever undertaken in imaging and spectroscopy (Stoughton et al. 2002) for hundreds of thousands galaxies. The imaging data are obtained in drift scan mode and are $95 \%$ complete for point sources at $22.0,22.2,22.2,21.3$, and 20.5 in five bands $(u, g, r, i, z)$, respectively. The spectroscopic data provide spectral flux and wavelength calibrated with 4096 pixels from 3800 to $9200 \AA$ at resolution $R \sim 1800$. Our sample was selected from the Main Galaxy Sample (MGS) of SDSS-DR7. Following Zhong et al. (2008), we firstly selected 21664 nearly face-on disk LSBGs from the SDSS-DR7 MGS. Then red and blue LSBGs were selected from their $g-r$ vs. $r-i$ diagram. These color-selected LSBGs were further matched with the spectral catalog to select those with higher signal-to-noise $(S / N)$ ratio on the spectral continua. The detailed selection criteria are given below.

1. fracDev $v_{r}<0.25$, indicating the fraction of luminosity contributed by the de Vaucouleurs profile relative to exponential profile in the $r$-band is much smaller (Bernardi et al. 2005; Chang et al. 2006; Shao et al. 2007); $b / a>0.75$, this corresponds to the inclination $i<41.41$ degree, which is to select the nearly face-on disk galaxies (Liu et al. 2009) ( $a$ and $b$ are the semi-major and semi-minor axes of the fitted exponential disk, respectively); $M_{B}<-18.0$, this is to exclude the few dwarf galaxies $\left(M_{B}\right.$ is the absolute magnitude in the $B$-band); $\mu_{0}(B) \geq 22.0 \mathrm{mag} \operatorname{arcsec}^{-2}$, this is to select the LSBGs (O'Neil et al. 1997; Impey et al. 2001; Boissier et al. 2003). After applying the above four selection criteria, 21664 nearly face-on disk LSBGs were selected. Their $\mu_{0}(B)$ are from 22.0 to 24.5 mag $\operatorname{arcsec}^{-2}$ with a median value of $22.43 \mathrm{mag} \operatorname{arcsec}^{-2}$.

2. In Fig-1a we present the histogram distribution of the redshift of this large sample of galaxies, showing $0.01<z<$ 0.27 with the median value of 0.08 . In Fig. $1 \mathrm{~b}$ we show the

\footnotetext{
1 http://wwW.sdss.org/DR7

2 http://www.starlight.ufsc.br
}

distributions of $g-r$ color with stellar mass (taken from the MPA/JHU stellar mass catalog as given in Kauffmann et al. 2003a; Gallazzi et al. 2005). It can be seen that there exists a slight correlation showing that more massive LSBGs have redder generally $g-r$ colors. In Fig. $1 \mathrm{c}, g-r$ color is plotted as a function of $D_{n}(4000)$ (taken from the MPA/JHU spectroscopic catalog as given in Kauffmann et al. 2003a). It shows a slight correlation between $g-r$ color and $D_{n}(4000)$ as well, showing that $g-r$ color becomes redder along with a higher value of $D_{n}(4000)$. Figure $1 \mathrm{~d}$ shows the $g-r$ versus $r-i$ diagram for the selected LSBGs.

Then two categories are selected from Fig. 1d: the blue LSBGs with $g-r<0.35, r-i<0.05$ (the bottom left corner of the solid lines and the red LSBGs with $g-r>0.6, r-i>$ 0.3 (the top right corner of the dashed lines). This step results in 405 red and 1,025 blue LSBGs.

3. Matching with the MPA/JHU ${ }^{3}$ spectroscopic catalog, 404 red and 1,022 blue LSBGs have spectral observations and $S / N$ measurements. To perform spectral synthesis studies on the galaxies through fitting their spectral continua and absorption lines, good $S / N$ are needed for their spectra. Therefore, we only selected the galaxies with median $S / N$ per pixel of the whole spectrum greater than 8.0. Then 226 red and 276 blue LSBGs are selected.

Figure 2 shows the histogram distribution of $S / N$ for all the 404 red (bottom) and 1,022 blue (top) LSBGs (with median values of 8.5 and 6.8 , respectively), and the selected sample with $S / N>8.0$ (226 red and 276 blue LSBGs) marked by the solid vertical lines (the right parts of the lines). Furthermore, we removed 13 red and 10 blue LSBGs, because their spectra are not continuous throughout the whole spectra due to some problems, which makes them unsuitable to be synthesized. Finally, we selected 213 red and 266 blue disk LSBGs to study their SFH and spectral synthesis as presented in Sects. 3 and 4, respectively. We call this sample the "T-sample" (T means total).

4. Furthermore, to minimize/check the effect of $B$-band surface brightness for the red LSBGs, we then selected a subsample by further considering the surface brightness limit $\mu_{0}(R) \geq 20.7 \mathrm{mag} \operatorname{arcsec}^{-2}$, which yielded 100 red and 262 blue LSBGs. This does show that the $B$-band surface brightness selection may benefit from also selecting blue LSBGs. However, we hope this will not have much affect on the basic results on the properties of these two types of galaxies with blue or red colors. We call this sub-sample the " $\mu$-sample", and will study its properties in Sect. 5.1.

5. For a more reliable comparison between red and blue LSBGs with a similar stellar mass, we further selected a sub-sample with a stellar mass limit of $9.5 \leq \log \left(M_{\star} / M_{\odot}\right) \leq 10.3$ from the total sample, which yielded 83 red and 120 blue LSBGs. We call this sub-sample "M-sample". Its properties will be specially presented in Sect. 5.2.

\section{Star formation history}

In this section we study the SFH of red and blue LSBGs with some property parameters, such as $\mathrm{Mg}_{2}, \mathrm{H} \delta_{A}$ (Worthey \& Ottaviani 1997), $D_{n}$ (4000) (Balogh et al. 1999), stellar mass, and surface mass density, the values of which are taken from or calculated on the basis of the MPA/JHU catalog.

$\mathrm{Mg}_{2}$, containing both $\mathrm{Mg} b$ and $\mathrm{MgH}$ absorption, is sensitive to metallicity and responds very similar like changes of

3 http://www . mpa-garching .mpg .de/SDSS/DR7 
The star formation histories of red and blue disk LSBGs: G.H. Zhong et al.
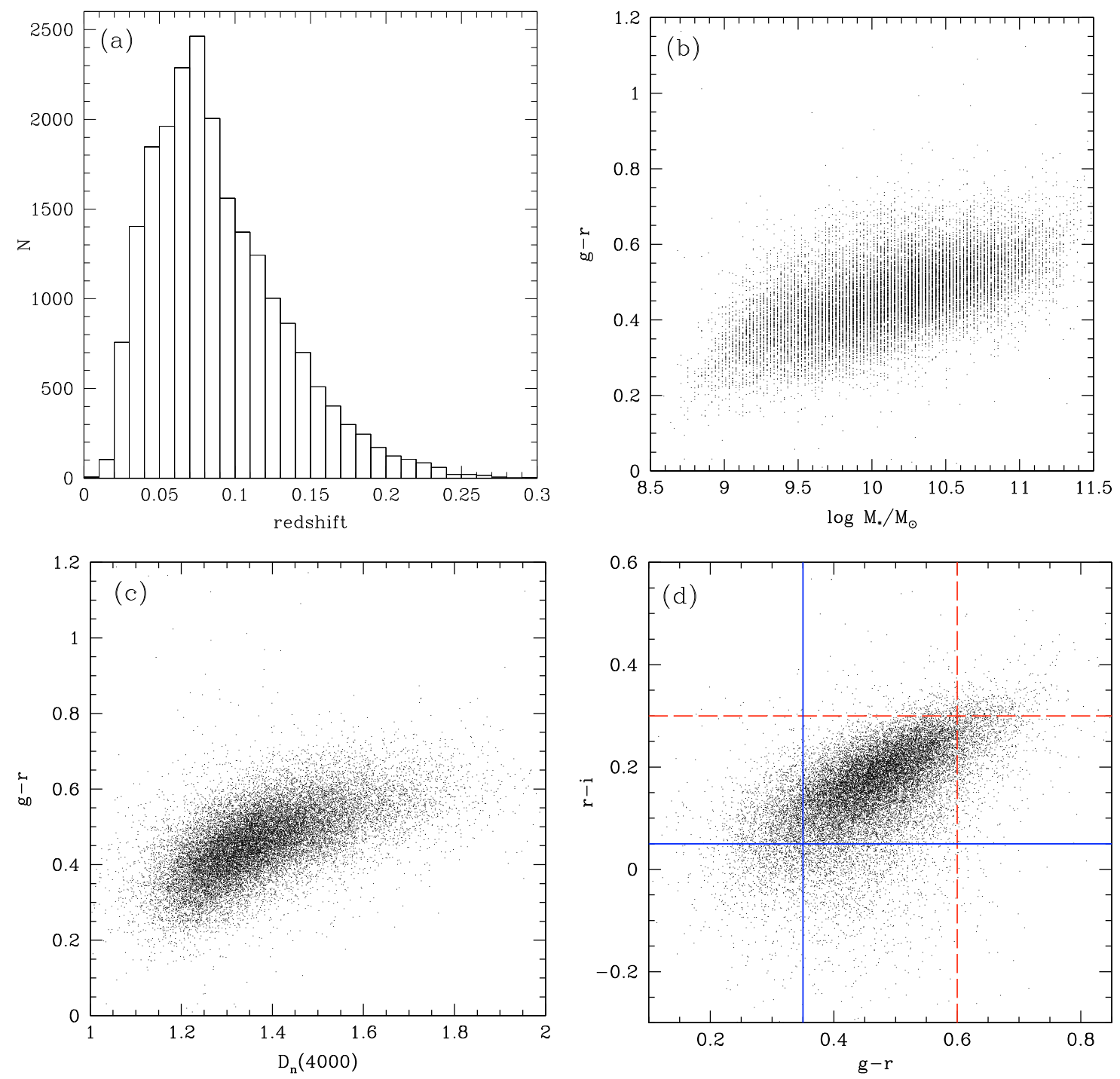

Fig. 1. The properties of the 21664 nearly face-on disk LSBGs: a) histogram distribution of redshift; b) distribution of $g-r$ colors with stellar mass; c) $g-r$ colors is plotted as a function of $D_{n}(4000)$; d) $g-r$ versus $r-i$ diagram, where the solid and dashed lines are used to define the red (top-right corner) and blue (bottom-left corner) LSBGs, respectively (see text).

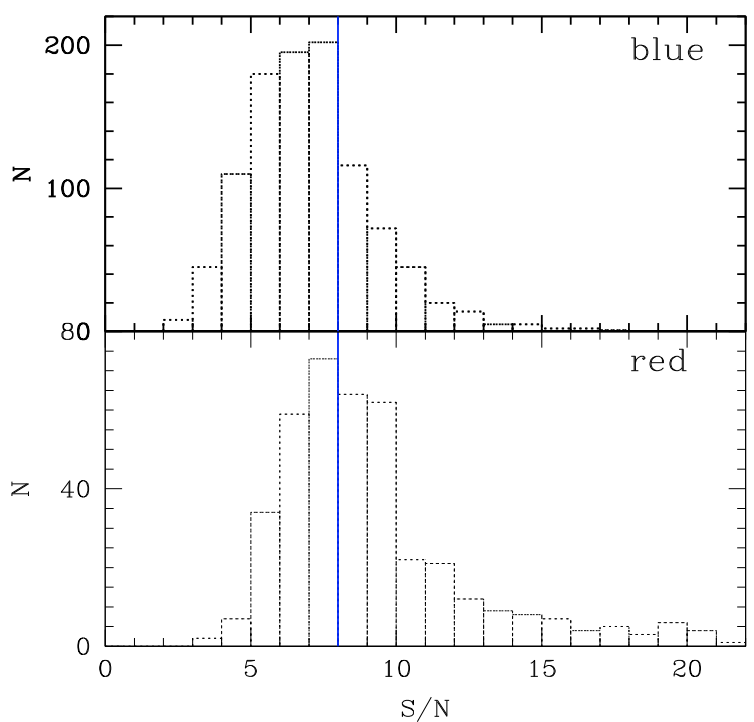

Fig. 2. Histogram distribution of $S / N$ for the 405 red (bottom) and 1,025 blue (top) LSBGs, respectively. The median values are 8.5 and 6.8 , respectively. The solid lines refer to $S / N=8.0$. $\alpha / \mathrm{Fe}$ to $\mathrm{Mg} b$ (Thomas et al. 2003). $\mathrm{Mg}_{2}$ increases with increasing $\alpha / \mathrm{Fe}$ ratio. The distributions of $\mathrm{Mg}_{2}$ with stellar mass are shown in Fig. 3a. It suggests that red LSBGs are generally more massive than the blue ones with the median values of $4.1 \times 10^{10} M_{\odot}$ and $7.6 \times 10^{9} M_{\odot}$, respectively. It is in accordance with the luminosity-metallicity relations that the redder colors correspond to galaxies with larger stellar mass (Galaz et al. 2002), meaning that more massive galaxies contain more metals and/or older stellar populations than lower mass galaxies, although with scatters.

It has been discussed by Kauffmann et al. (2003a) that $D_{n}(4000)-\mathrm{H} \delta_{A}$ plane is a powerful diagnostic for the SFH of galaxies that shows e.g. whether galaxies have been forming stars continuously or in bursts over the past 1-2 Gyr. Therefore, we use the narrow definition of $D_{n}(4000)$ and $\mathrm{H} \delta_{A}$ as a diagnostic of the SFH of red and blue LSBGs. In Fig. 3b we show the $\mathrm{H} \delta_{A}$ absorption index as a function of $D_{n}(4000)$. One can clearly see that nearly all blue LSBGs have lower $D_{n}(4000)$ values $(\leq 1.4$, characteristic of stellar populations with mean ages of less than a few Gyr, Kauffmann et al. 2003b) and stronger $\mathrm{H} \delta_{A}$ absorption than red LSBGs. It means that blue LSBGs have a higher fraction of young stars, hence have smaller mass-to-light 

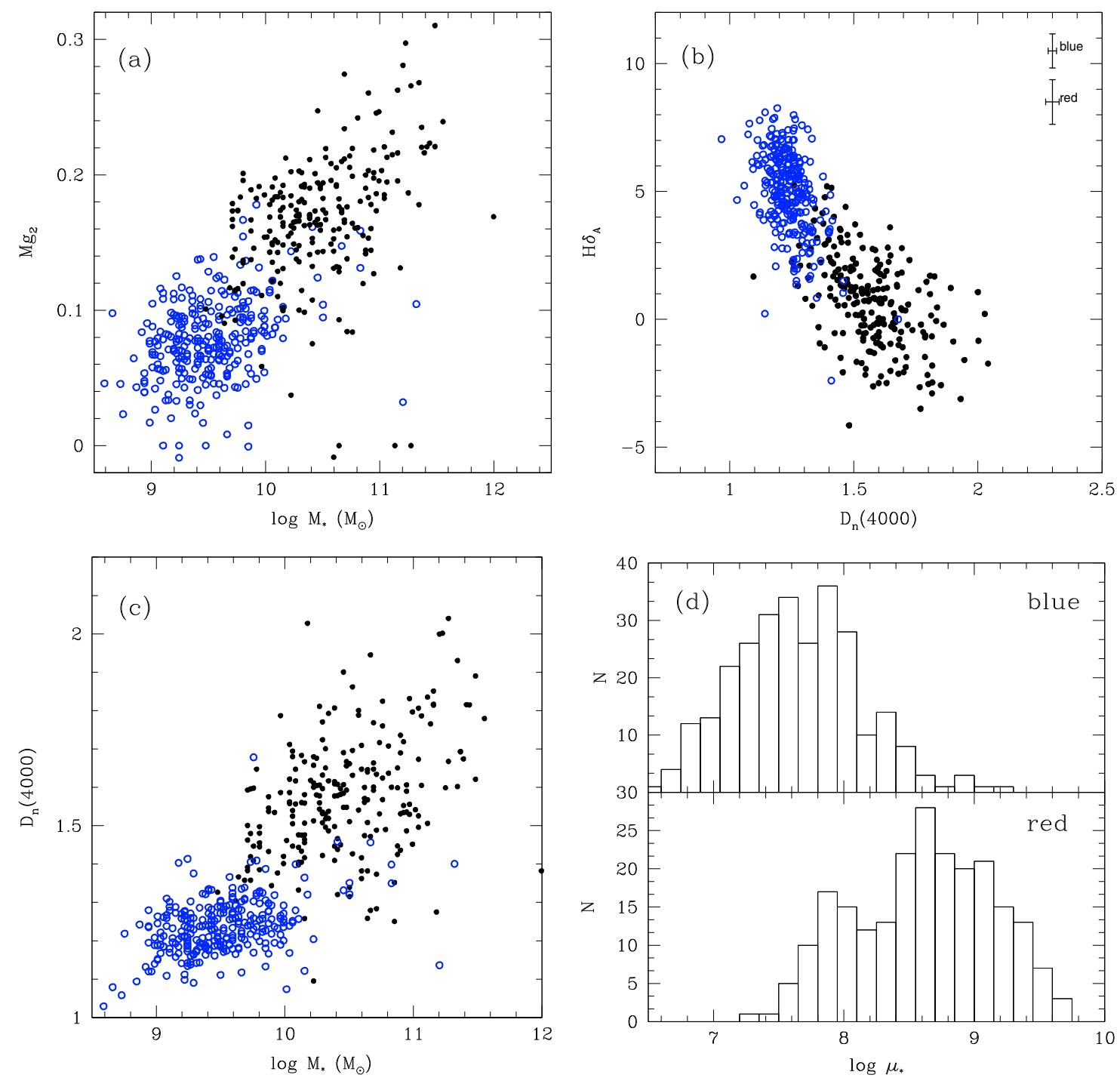

Fig. 3. The properties of red and blue LSBGs: a) the distribution of $\mathrm{Mg}_{2}$ as a function of stellar mass; $\left.\mathbf{b}\right) \mathrm{H} \delta_{A}$ is plotted as a function of $D_{n}(4000)$; c) the relation between $D_{n}(4000)$ and stellar mass; d) histogram distribution of surface mass densities for red (bottom) and blue (top) LSBGs. The filled and open circles denote red and blue LSBGs, respectively.

ratios (in $z$ band, Kauffmann et al. 2003a), and are more likely to be experiencing a sporadic star formation event at the present day. Whereas red LSBGs are distributed in the continual star formation regions suggested by Kauffmann et al. (2003a, see their Fig. 6), which means that red LSBGs are more likely to form stars continually over the past years. This may be due to their higher stellar mass and corresponding higher surface mass density (Kauffmann et al. 2003b). Moreover, the $\mathrm{H} \delta_{A}$ of blue LSBGs drops more rapidly than red LSBGs with the increasing $D_{n}(4000)$.

In Fig. 3c $D_{n}(4000)$ is plotted as a function of stellar mass. Considering Figs. $3 b$ and $3 c$ together, we notice that the fraction of galaxies that have experienced recent sporadic star formation events decreases with increasing stellar mass, which is consistent with Kauffmann et al. (2003b, see their Fig. 3).

In Fig. 3d we show the histogram distribution of surface mass densities for red (bottom) and blue (top) LSBGs. Following Kauffmann et al. (2003b), we define the surface mass density $\mu_{\star}$ as $0.5 M_{\star} /\left[\pi R_{50}^{2}(z)\right]$, where $R_{50}(z)$ is the Petrosian half-light radius in the $z$ band. The surface mass density of red LSBGs is higher than blue LSBGs, with median values $4.0 \times 10^{8}$ and $4.0 \times 10^{7}$ for red and blue LSBGs, respectively.
It is suggested that galaxies with larger stellar masses tend to have higher surface mass density (Kauffmann et al. 2003b), but smaller gas mass fractions (Galaz et al. 2002). Thus the older stellar populations and higher surface mass density of red LSBGs indicate an epoch of more vigorous star formation over the past years, which has also been suggested by Bell et al. (1999). The younger stellar populations and lower mean metallicities of blue LSBGs principally indicate the galaxies are being slow to convert gas into stars (Bell et al. 2000; Schombert et al. 2001) and are relatively unevolved.

\section{Spectral synthesis}

Spectral synthesis provides a new way to retrieve information of stellar populations of galaxies from observational spectra, which is crucial for a deeper understanding of galaxy formation and evolution. Galaxy spectra contain information about the age and metallicity distributions of the stars, which in turn reflects the star formation and chemical enrichment histories of the galaxies.

We fitted the optical spectra of red and blue LSBGs with the spectra synthesis code STARLIGHT (Cid Fernandes et al. 2005; Mateus et al. 2006; Asari et al. 2007). The method consists of 
The star formation histories of red and blue disk LSBGs: G.H. Zhong et al.
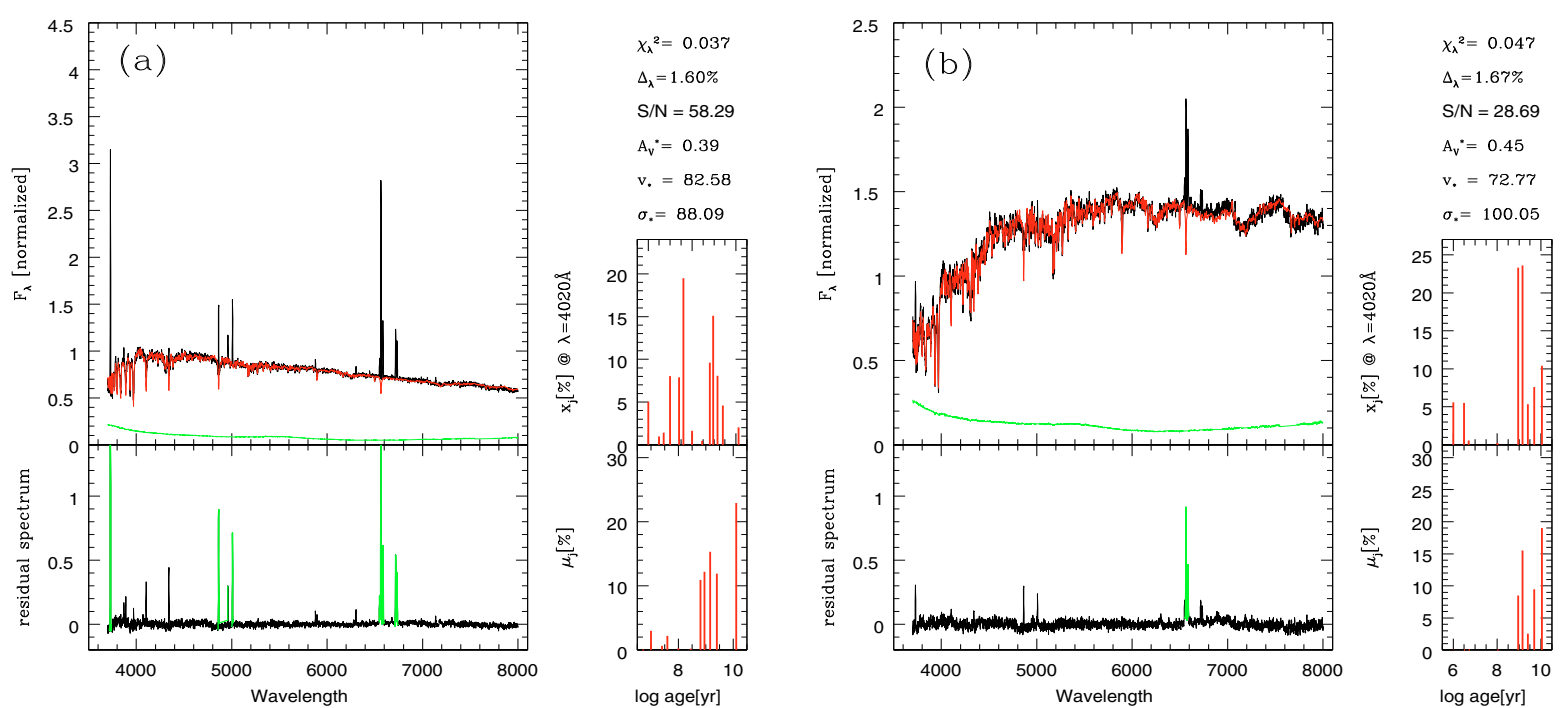

Fig. 4. Combined spectral synthesis results of blue (left) and red (right) LSBGs by using STARLIGHT with 45 SSPs from Bruzual \& Charlot (2003). In each of the panels, top left: the synthesis spectrum (red line), the observed spectrum (black line), and the error spectrum (green line); bottom left: the residual spectrum, the green lines represent masked regions given by the SDSS flag; right: the contribution fractions of light (top) and mass (bottom) as a function of the 15 ages of SSPs. We list six parameters in the top right corners. (Please see on-line color version for more details.)

Table 1. Stellar populations of red and blue disk LSBGs.

\begin{tabular}{cccc}
\hline \hline & SSP & blue LSBGs & red LSBGs \\
\hline age $(t$, Gyr $)$ & young $(t \leq 0.5)$ & 58.8 & 38.9 \\
& intermediate $(0.64 \leq t \leq 2.5)$ & 35.3 & 27.8 \\
& old $(t \geq 5.0)$ & 5.9 & 33.3 \\
\hline$Z / Z_{\odot}$ & 0.2 & 58.8 & 35.3 \\
& 1 & 29.4 & 47.1 \\
& 2.5 & 11.8 & 17.6 \\
\hline
\end{tabular}

Notes. Results of fitting the combined spectra by using STARLIGHT with 45 SSPs from Bruzual \& Charlot (2003). The contributed light fractions in three age-bins (young populations with age $\leq 5 \times 10^{8} \mathrm{yr}$, intermediate populations with $6.4 \times 10^{8} \mathrm{yr} \leq$ age $\leq 2.5 \times 10^{9} \mathrm{yr}$ and old populations with age $\left.\geq 5.0 \times 10^{9} \mathrm{yr}\right)$ and three metallicities $\left(0.2,1.0\right.$ and $\left.2.5 Z_{\odot}\right)$ are presented. It is noticed that the percent fractions here are slightly different from the $x_{j}$ component output by STARLIGHT directly.

fitting an observed spectrum $O_{\lambda}$ with a model $M_{\lambda}$ combination of $N_{*}$ spectral components SSPs taken from Bruzual \& Charlot (2003). In this work, we take 45 SSPs, including 15 different ages from 1 Myr to $13 \mathrm{Gyr}$ (i.e. 1, 3, 5, 10, 25, 40, 100, 280, 640, $900 \mathrm{Myr}$ and 1.4, 2.5, 5, 11, $13 \mathrm{Gyr}$ ) and three metallicities (i.e. $0.2,1$, and $2.5 Z_{\odot}$ ), the stellar evolutionary tracks of Padova 1994 (Alongi et al. 1993; Girardi et al. 1996), the initial mass function (IMF) of Chabrier (2003), and the extinction law of Cardelli et al. (1989) with $R_{V}=3.1$. The Galactic extinctions are corrected by the reddening map of Schlegel et al. (1998), then shifted to the rest frame. The range of the spectra is from 3700 to $8000 \AA$ in step of $1 \AA$ and normalized by the median flux in the 4010 to $4060 \AA$ region by the median value. During spectral synthesis fitting, we exclude the emission lines, sky lines and another four windows (5870-5905 $\AA$, 6845-6945 ̊,7550-7725, $7165-7210 \AA$ ) as done in Chen et al. (2009, 2010).

Figure 4 shows the spectral fitting results of the combined spectra for blue (left) and red (right) LSBGs. There are four sub-panels in each panel: the top left shows the synthesis spectrum (red line), the observed spectrum (black line), and the error spectrum (green line); bottom left shows the residual spectrum, the green lines represent mask regions given by SDSS flag; the right panel shows the contributed fractions to light (top) and mass (bottom) from the 15 SSPs with different ages.
We list the produced six parameters in the top right corners, such as $\chi_{\lambda}^{2}$, i.e. the reduced $\chi^{2}$; the mean relative difference between synthesis and observed spectra $\Delta_{\lambda}$; the $S / N$ in the region of 4730-4780 $\AA$; $V$-band extinction; the velocity $v_{\star}$ and the velocity dispersion $\sigma_{\star}$. The contributed light fractions of the stellar populations in age-bin and metallicity-bin are presented in Table 1 . The three age bins are young populations with age $\leq 5 \times 10^{8} \mathrm{yr}$, intermediate populations with $6.4 \times 10^{8} \mathrm{yr} \leq$ age $\leq$ $2.5 \times 10^{9} \mathrm{yr}$ and old populations with age $\geq 5.0 \times 10^{9} \mathrm{yr}$ and their metallicities are $0.2,1.0$ and $2.5 Z_{\odot}$, respectively.

Figure 4 and Table 1 show that red LSBGs are older than blue LSBGs. Blue LSBGs are dominated by the young $(58.8 \%)$ and intermediate age populations $(35.3 \%)$ with a small fraction of old age populations ( $5.9 \%$ ). Red LSBGs contain a very significant fraction of old age populations $(33.3 \%$, about $27 \%$ larger than blue ones), although they also have significant young age populations $(38.9 \%)$, which suggests that there was an epoch of more vigorous star formation in red LSBGs in the past. This is consistent with Bell et al. (1999), who commented that red LSBGs have higher mean stellar ages than blue LSBGs.

It also shows that the metallicities of red LSBGs are higher than that of blue LSBGs, but, the difference of the metallicities between red and blue LSBGs is not as obvious as the difference of the age populations. The dominant metallicities are $Z_{\odot}$ for red $(47.1 \%)$ and $0.2 Z_{\odot}$ for blue $(58.8 \%)$ LSBGs. The fraction of 

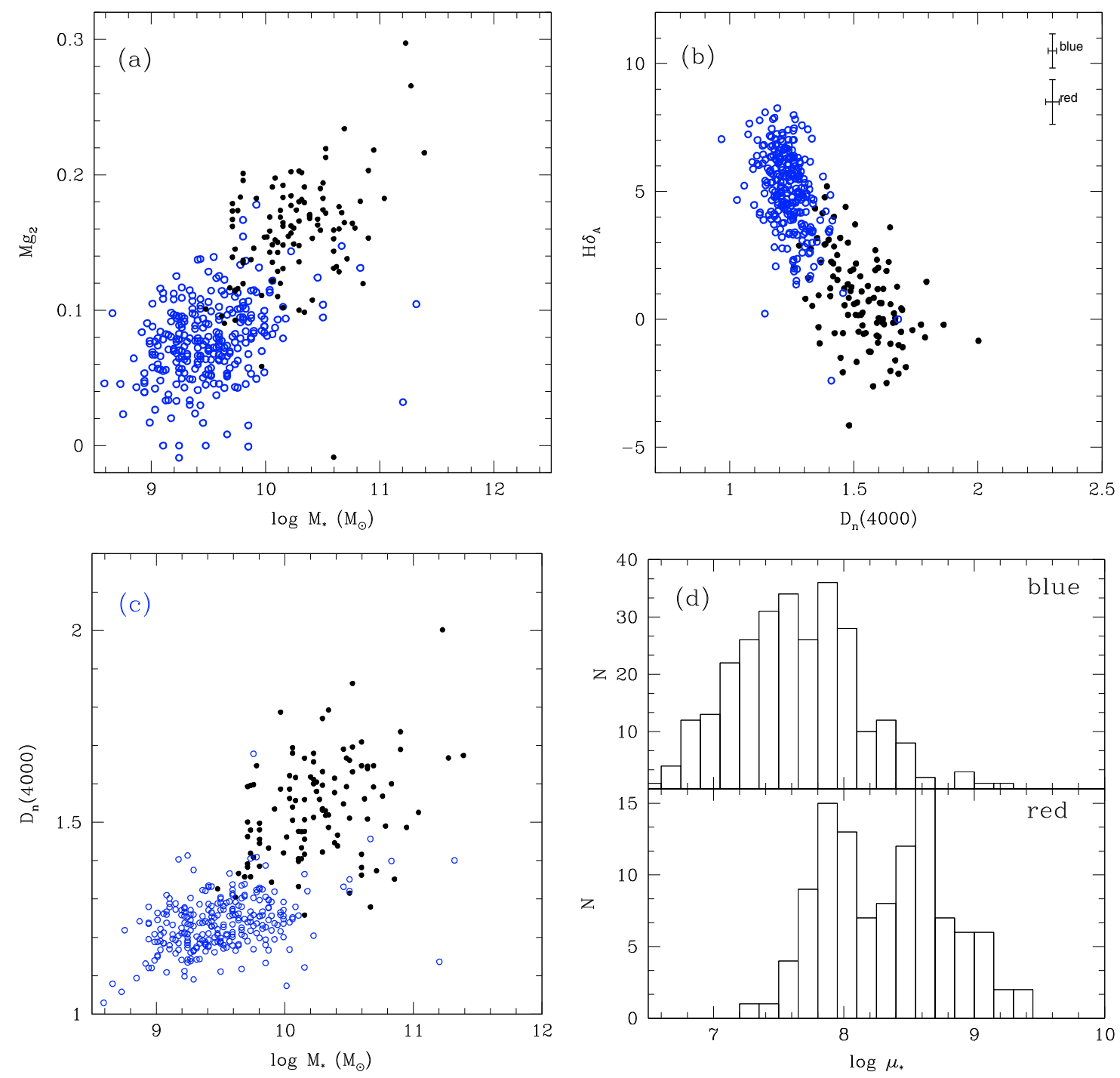

Fig. 5. The same as Fig. 3 but for the surface brightness limiting sub-samples further with $\mu_{0}(R) \geq 20.7$ mag $\operatorname{arcsec}^{-2}$.

metallicities of $2.5 Z_{\odot}$ in red LSBGs are $17.6 \%$, which is $6 \%$ higher than blue LSBGs $(11.8 \%)$. It should be noticed that the dominant contributions of stellar mass are all old in both groups. We will discuss the uncertainties of these results in Sect. 6, but, we believe that they are insignificant.

\section{The sub-samples}

In this section, we select two sub-samples, the surface brightness limiting sub-sample ( $\mu$-sample) and the mass limiting subsample (M-sample), to discuss the selection effects of surface brightness and stellar mass.

\subsection{Surface brightness limiting sub-sample}

Our selection criterion of LSBGs is $\mu_{0}(B)$, which is a blue-filter surface brightness criterion, thus some red LSBGs may not be intrinsically of low surface brightness. Therefore, we further define a second criterion to limit the surface brightness in both the blue and red filters by adding the $\mu_{0}(R)$ selection criterion to our total sample. Courteau (1996) found there is a well-defined upper cutoff at $\mu_{0}(R)=20.08 \pm 0.55 \mathrm{mag} \operatorname{arcsec}^{-2}$ (see also the introduction of Galaz et al. 2002) for LSBGs. We chose
$\mu_{0}(R) \geq 20.7 \mathrm{mag} \operatorname{arcsec}^{-2}$ as our red filter selection criterion and then obtained 100 red and 262 blue LSBGs as our surface brightness limiting sub-sample, the $\mu$-sample. Following Fig. 3 and Table 1, their properties are shown in Fig. 5, and the results of spectral synthesis are shown in Table 2.

The results show that red LSBGs are much affected by the red-filter selection criterion, and 113 red LSBGs were removed because of this. Only four blue LSBGs were removed by this criterion. Therefore, the distributions of red and blue LSBGs show some differences in the $\mathrm{Mg}_{2}$ vs. $\log \left(M_{\star} / M_{\odot}\right)$ (Fig. 5a) and $D_{n}(4000)$ vs. $\log \left(M_{\star} / M_{\odot}\right)$ (Fig. 5c) relations. Comparing Figs. $3 \mathrm{~d}$ and $5 \mathrm{~d}$, we can see that the samples with a relatively higher surface mass density are removed. However the median surface mass density values of red and blue LSBGs are $2.5 \times 10^{8}$ and $4.0 \times 10^{7}$, respectively, which is not so different from the total sample $\left(4.0 \times 10^{8}\right.$ and $4.0 \times 10^{7}$, respectively). The distributions of the sub-sample galaxies in the $D_{n}(4000)-\mathrm{H} \delta_{A}$ plane (Fig. $5 \mathrm{~b}$ ) also do not show much difference from the total sample as given in Fig. 3b. Comparing Tables 2 and 1, it shows that this $\mu_{0}(R)$ limit nearly does not change the fraction of stellar populations of blue LSBGs, because only four blue LSBGs are removed. For red LSBGs, the $\mu_{0}(R)$ limit increases the intermediate stellar population by $11 \%$ and correspondingly decreases the old stellar population by $11 \%$, and the young stellar population show nearly 
The star formation histories of red and blue disk LSBGs: G.H. Zhong et al.
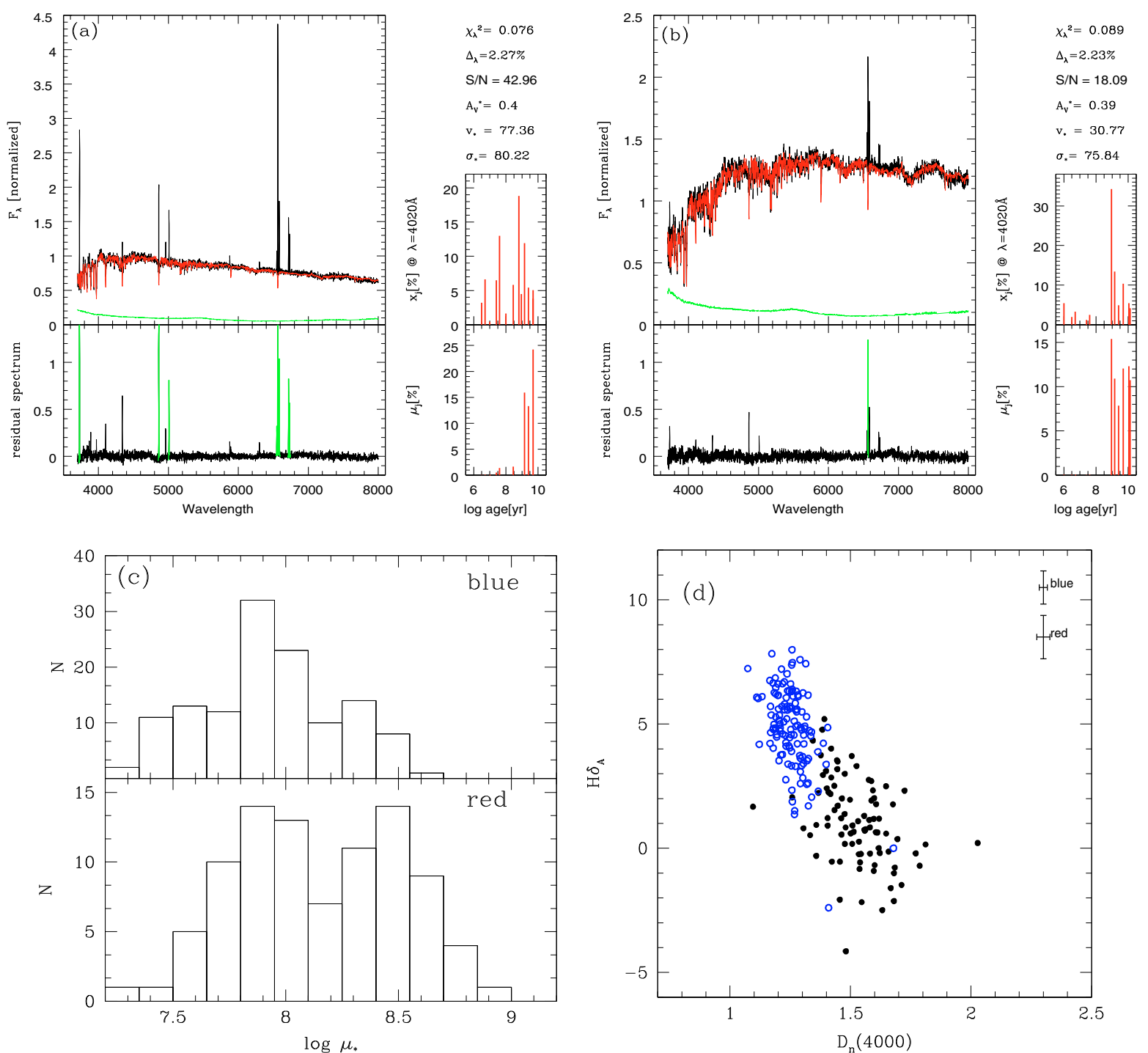

Fig. 6. The properties of mass limiting sub-samples for red and blue LSBGs: a) the same as Fig. 4, but for the sub-sample of blue LSBGs; b) the same as Fig. 4 for the sub-sample of blue LSBGs; c) histogram distribution of surface mass densities for the sub-samples of red (bottom) and blue (top) LSBGs; d) the relations between $D_{n}(4000)$ and stellar mass for sub-samples.

Table 2. Stellar populations of red and blue LSBGs sub-samples for surface brightness limiting with $\mu_{0}(R) \geq 20.7$ mag arcsec $^{-2}$ and stellar mass limiting with $9.5 \leq \log \left(M_{\star} / M_{\odot}\right) \leq 10.3$.

\begin{tabular}{cccccc}
\hline \hline & SSPs & surface brightness limiting sub-samples & stellar mass limiting sub-samples \\
\hline & & blue LSBGs & red LSBGs & blue LSBGs & red LSBGs \\
\hline age $(t$, Gyr $)$ & young $(t \leq 0.5)$ & 61.9 & 38.9 & 60.0 & 41.7 \\
& intermediate $(0.64 \leq t \leq 2.5)$ & 33.3 & 38.9 & 35.0 & 25.0 \\
& old $(t \geq 5.0)$ & 4.8 & 22.2 & 5.0 & 33.3 \\
\hline$Z / Z_{\odot}$ & 0.2 & 42.9 & 44.5 & 60.0 & 41.6 \\
& 1 & 33.3 & 22.2 & 25.0 & 29.2 \\
& 2.5 & 23.8 & 33.3 & 15.0 & 29.2 \\
\hline
\end{tabular}

no changes. This means that this $\mu_{0}(R)$ limit removes some red LSBGs with older stellar populations.

\subsection{Mass limiting sub-sample}

In order to get a fair comparison between red and blue LSBGs, we selected two mass limiting sub-samples of red and blue LSBGs from our total sample (rather than the surface brightness limiting sub-sample with $\mu_{0}(R)$, because if we were to select mass limiting sub-samples from the surface brightness limiting sub-sample, the sources of red LSBGs would be very small. Considering the overlap of stellar mass in Figs. $3 \mathrm{~b}$ and $3 \mathrm{c}$ between red and blue LSBGs, we defined the range of $9.5 \leq$ $\log \left(M_{\star} / M_{\odot}\right) \leq 10.3$ for both red and blue LSBGs. We obtained 83 red and 120 blue LSBGs in this stellar mass range as our two mass limiting sub-samples for more comparisons.

In Fig. 6, we show the properties of the two sub-samples of red and blue LSBGs. Figures $6 \mathrm{a}$ and $6 \mathrm{~b}$ are the same as Figs. $4 \mathrm{a}$ 
and $4 \mathrm{~b}$, but for the two mass limiting sub-samples. Table 2 (right part) presents the contributed light fractions of the stellar populations to the blue and red LSBGs. They are very similar to those of the total sample as given in Table 1, less than 3\% discrepancy in the stellar populations with age bins. Figure $6 \mathrm{c}$ shows the histogram distribution of surface mass densities for our mass limiting sub-sample. Red LSBGs also have surface mass densities a little higher than blue LSBGs, with median values of $1.6 \times 10^{8}$ and $7.4 \times 10^{7}$, respectively. These are also similar to the total sample, and to the surface brightness limiting sub-sample as well. Moreover, these mass limiting sub-samples also do not show much difference in the distribution of the $D_{n}(4000)-\mathrm{H} \delta_{A}$ plane, just with less scatter (Fig. 6d). This also confirms that the SFH properties are different between red and blue LSBGs, as Fig. 4d showed.

\section{Discussions}

We discuss the uncertainties of spectral synthesis and the aperture effects in this section.

\subsection{Uncertainties of spectral synthesis}

We discuss the uncertainties of the stellar populations of galaxies calculated with the STARLIGHT code and the SSP templets from Bruzual \& Charlot (2003). In our earlier work Chen et al. (2010), we have also discussed the uncertainties of the resulted stellar populations by using STARLIGHT to compare six popular evolutionary stellar population synthesis models.

As we know, the STARLIGHT group has used this code to analyze a large sample of SDSS galaxies as shown in their series of work (Cid Fernandes et al. 2005, 2007; Mateus et al. 2006; Asari et al. 2007). And they have tested the uncertainties of the resulted stellar populations. In the study of Cid Fernandes et al. (2005), they found that uncertainties are less than $0.05,0.1$, and 0.1 for young $\left(t<10^{8}\right)$, intermediate $\left(10^{8}<t<10^{9}\right)$, and old $\left(t>10^{9}\right)$ populations for $S / N \geq 10$, respectively. In our fittings on the sample galaxies, the code provides the values (e.g. the contributed light fractions of SSPs) of last-chain-values for seven Markov chains, and we find that most of the discrepancies in these adopted values are less than $1 \%$. Thus we believe the uncertainties of the resulted light fractions are small, and we consider the uncertainties of the resulting stellar populations to be insignificant.

Most of the age sensitivity comes from the continuum shape and $D_{n}(4000)$ break in the fittings, thus degeneracy with dust should be discussed carefully. The dust extinction has been considered in STARLIGHT as a variable parameter, and we adopt the extinction law of Cardelli et al. (1989) for the code. The resulting dust extinction $A_{V}$ from the code is around 0.4 for the sample galaxies as given in Figs. 4 and 6. We also measured the $A_{V}$ value from $\mathrm{H} \alpha / \mathrm{H} \beta$ for the blue LSBGs from the combined spectra as given in Fig. 4a. It is just about 0.4 , consistent with the resulting from the STARLIGHT program. Thus, the dust extinction effect could have been reliably considered in the the stellar population analyses here.

Furthermore, we used a simpler method to test the age and metallicity degeneracy in the resulted stellar populations from STARLIGHT. We use $15 \mathrm{SSPs}$ with 15 ages at $Z=Z_{\odot}$ to do the spectral synthesis on the blue and red LSBGs, and then use another $15 \mathrm{SSPs}$ with same ages but at $Z=0.2 Z_{\odot}$ to re-do the spectral synthesis. Comparing these results with those from all three $Z$ cases (Table 1 ), the $Z=Z_{\odot}$ results show $\sim 12 \%$ more

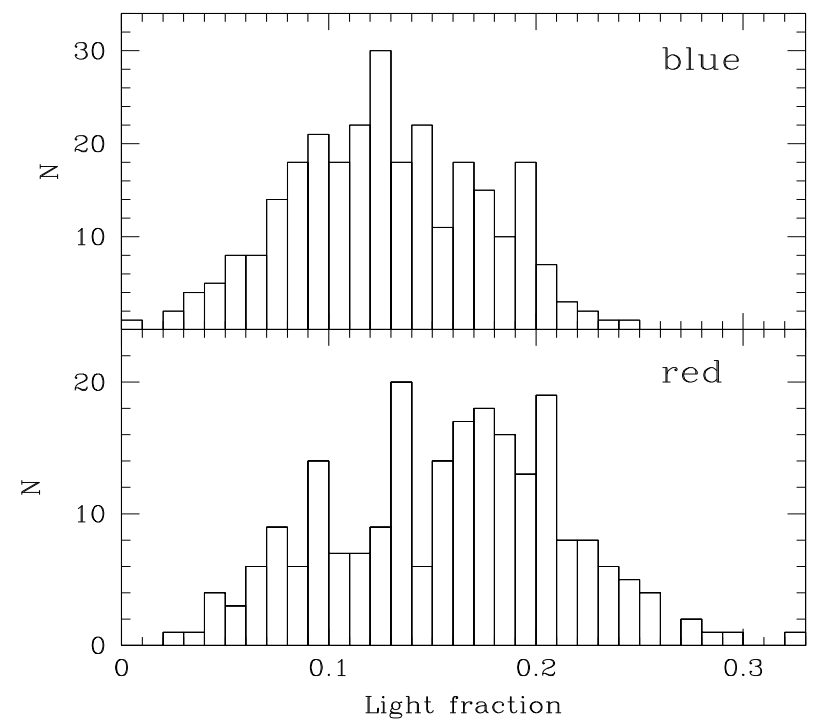

Fig. 7. Histogram distributions of the light fraction (Eq. (1)) with bins of 0.01 for both red (bottom) and blue (top) LSBGs of our total sample. The median values of the light fractions are 0.16 for red LSBGs and 0.13 for blue LSBGs.

young population for blue LSBGs, and $~ 7 \%$ less intermediate populations; and the $Z=0.2 Z_{\odot}$ results show $\sim 9 \%$ less young populations, and $\sim 10 \%$ more old populations; for red LSBGs, the light fractions show few changes. Thus, from these analysis the metallicity will cause uncertainties of about $10 \%$ for the stellar populations of blue LSBGs, and have a very small effect on red LSBGs. But here we prefer the three metallicity results. In any case, the dominant population of the sample galaxies are unchanged, i.e. the young population.

\subsection{Aperture effects}

The SDSS is a fiber-based survey. Consequently, we consider the aperture effects in this section. Tremonti et al. (2004) and Kewley et al. (2005) have discussed the weak effect of the $3^{\prime \prime}$ aperture of SDSS spectroscopy. They believe that redshifts $z>0.03$ and 0.04 respectively are required for SDSS galaxies to get reliable metallicities. It is agreed that the fiber mag is a measurement of the light going down the fiber and the petrosian mag is a good estimate of the total magnitude. Thus, to check how much the light of the galaxies was covered by the fiber observation, one simple and accurate way is to compare the "fiber" and "Petrosian" magnitudes of the SDSS galaxies (Liang et al. 2010). We choose the formula:

light_fraction $=10^{\left(-0.4 \times(\text { fiber_mag-petro_mag })_{r}\right)}$

to estimate how much light was covered by the fiber observations. Figure 7 shows the light fractions for red and blue LSBGs of our total sample. It shows that the light fractions of red (bottom) and blue (top) LSBGs are 0.16 and 0.13 , respectively. Therefore, the properties of red and blue LSBGs stem mainly from the central regions, which may be redder, older and more metal-rich than the outer regions. However, it could not much affect our results of the differences between red and blue LSBGs, because the light fractions are almost the same. Moreover, all our sample galaxies are disk-dominated galaxies with very small bulges, so we assume the resulted stellar populations could be good representatives of their disk populations. 
The star formation histories of red and blue disk LSBGs: G.H. Zhong et al.

\section{Summary}

Our main results can be summarized as follows. We present a large sample of 213 red and 266 blue disk LSBGs from SDSSDR7, which have sufficient $S / N$ in the spectral continua to study their SFH though spectral synthesis with the STARLIGHT code and the SSPs of Bruzual \& Charlot (2003), as well as the absorption-line indices $\left(\mathrm{Mg}_{2}, \mathrm{H} \delta_{A}\right)$ and $D_{n}(4000)$.

1. Blue LSBGs are dominated by young populations with a few old populations (5.9\% populations older than $5 \mathrm{Gyr}$ ), but, there is a significant fraction of old populations in red LSBGs $(33.3 \%)$. The dominant populations of blue LSBGs are $Z=0.2 Z_{\odot}$, while the dominant populations of red LSBGs are $Z=Z_{\odot}$, and red LSBGs are more metal-rich. Red LSBGs tend to be more massive and have a higher surface mass density than blue LSBGs.

2. The $D_{n}(4000)-\mathrm{H} \delta_{A}$ plane shows that red LSBGs have a different SFH from blue LSBGs: blue LSBGs are more likely to be experiencing a sporadic star formation event now, whereas red LSBGs are more likely to form stars continuously. Moreover, the fraction of galaxies that experienced recent sporadic star formation events deceases with increasing stellar mass.

3. By defining two sub-samples according to surface brightness $\mu_{0}(R)$ and stellar mass limits for both blue and red LSBGs, i.e. the $\mu$-sample with $\mu_{0}(R) \geq 20.7 \mathrm{mag} \mathrm{arcsec}^{-2}$, and the M-sample with $9.5 \leq \log \left(M_{\star} / M_{\odot}\right) \leq 10.3$, we find that they show very similar results to the total sample (T-sample) on the $D_{n}(4000)-\mathrm{H} \delta_{A}$ plane, surface mass density and stellar populations etc. This is a good confirmation of our comparisons for blue and red LSBGs.

The Large Synoptic Survey Telescope (LSST Science Collaborations 2009) will be sensitive to galaxies with central surface brightness as low as $27 \mathrm{mag} \mathrm{arcsec}^{-2}$ in the $r$-band in the ten-year stack-compared with SDSS, where the faintest galaxies measured have a central surface brightness $\mu_{r} \sim 24.5 \mathrm{mag} \mathrm{arcsec}^{-2}$ (Zhong et al. 2008). Moreover, with this aspect we will also discover larger numbers of giant LSBGs spirals and tie down the population of red spiral LSBGs. Therefore it is helpful to study the stellar populations and SFH from the LSST data sets in the future.

Acknowledgements. We thank the referee for the valuable comments which helped to improve this work. We thank Dr. James Wicker for helping us to correct the English. This work was supported by the NSFC grants 10933001 , 10973015, 10673002, and the National Basic Research Program of China (973 Program) grants 2007CB815404, 2007CB815406, and No. 2006AA01A120 (863 project). This work was supported by the Young Researcher Grant of National Astronomical Observatories, Chinese Academy of Sciences. The STARLIGHT project is supported by the Brazilian agencies CNPq, CAPES, and FAPESP and by the France-Brazil CAPES/Cofecub program. We thank the useful SDSS database and the MPA/JHU catalogs.

\section{References}

Abazajian, K., Adelman-McCarthy, J. K., Agüeros, M. A., et al. 2009, ApJS, 182,543
Adelman-McCarthy, J. K., Agüeros, M. A., Allam, S. S., et al. 2006, ApJS, 162, 38

Alongi, M., Bertelli, G., Chiosi, C., et al. 1993, A\&AS, 97, 851

Asari, N. V., Cid Fernandes, R., Stasinska, G., et al. 2007, MNRAS, 381, 263

Balogh, M. L., Morris, S. L., Yee, H. K. C., Carlberg, R. G., \& Ellingson, E. 1999, ApJ, 527, 54

Bell, E. F., Bower, R. G., de Jong, R. S., Hereld, M., \& Rauscher, B. J. 1999, MNRAS, 302, L55

Bell, E. F., Barnaby, D., Bower, R. G., et al. 2000, MNRAS, 312, 470

Bernardi, M., Sheth, R. K., Nichol, R. C., Schneider, D. P., \& Brinkmann J. 2005, AJ, 129, 61

Blanton, M. R., Brinkmann, J., Csabai, I., et al. 2003, AJ, 125, 2348

Boissier, S., Monnier-Ragaigne, D., Prantzos, N., et al. 2003, MNRAS, 343, 653

Bruzual, A. G., \& Charlot, S. 2003, MNRAS, 344, 1000

Burkholder, V., Imepy, C., \& Sprayberry, D. 2001, AJ, 122, 2318

Cardelli, J. A., Clayton, G., \& Mathis, J. 1989, ApJ, 345, 245

Chabrier, G. 2003, PASP, 115, 763

Chang, R. X., Shen, S. Y., Hou, J. L., Shu, C. G., \& Shao, Z. Y. 2006, MNRAS, 372,199

Chen, X. Y., Liang, Y. C., Hammer, F., Zhao, Y. H., \& Zhong, G. H. 2009, A\&A, 495,457

Chen, X. Y., Liang, Y. C., Hammer, F., et al. 2010, A\&A, 515, A101

Cid Fernandes, R. 2007, in press [arXiv:astro-ph/0701902]

Cid Fernandes, R., Mateus, A., \& Sodre, L. 2005, MNRAS, 358, 363

Courteau, S. 1996, ApJS, 103, 363

de Blok, W. J. G., \& McGaugh, S. S. 1997, MNRAS, 290, 533

Galaz, G., Dalcanton, J., Infante, L., \& Treister, E. 2002, AJ, 124, 1360

Gallazzi A., Charlot S., Brinchmann J., White S. D. M., \& Tremonti C. 2005, MNRAS, 362, 41

Girardi, L., Bressan, A., Chiosi, C., Bertelli, G., \& Nasi, E. 1996, A\&AS, 117, 113

Haberzettl, L., Bomans, D. J., \& Detmar, R. J. 2007, A\&A, 471, 787

Impey, C. D., Burkholder, V., \& Sprayberry, D. 2001, AJ, 122, 2341

Kauffmann, G., Heckman, T. M., White, S. D. M., et al. 2003a, MNRAS, 341, 33

Kauffmann, G., Heckman, T. M., White, S. D. M., et al. 2003b, MNRAS, 341, 54

Kewley, L. J., Jansen, R. A., \& Geller, M. J. 2005, PASP, 117, 227

Liang, Y. C., et al. 2010, MNRAS, in press [arXiv: 1004.3683]

Liu, C. Z., Shen, S. Y., Shao, Z. Y., et al. 2009, RAA, 9, 1119

LSST Science Collaborations: Abell, P. A., et al. 2009, preprint [arXiv:0912.0201]

MacLow, M. M., \& Ferrera, A. 1999, ApJ, 513, 15

Mateus, A., Sodre, L., Cid Fernandes, R., et al. 2006, MNRAS, 370, 721

McGaugh, S. S., \& de Blok, W. J. G. 1997, ApJ, 481, 689

McGaugh, S. S., Bothun, G. D., \& Schombert, H. M. 1995, AJ, 110, 573

O’Neil, K., \& Bothun, G. 2000, ApJ, 529, 811, 136

O’Neil, K., Bothun, G., Schombert, J. M., Cornell, M. E., \& Impey, C. D. 1997, AJ, 114, 2448

O’Neil, K., Oey, M. S., \& Bothun, G. 2007, AJ, 134, 547

Rosenbaum, S. D., Krusch, E., Bomans, D. J., \& Dettmar, R. J. 2009, A\&A, 504, 807

Schlegel, D., Finkbeiner, D., \& Davis, M. 1998, ApJ, 500, 525

Schombert, J. M., McGaugh, S. S., \& Eder, J. A. 2001, AJ, 121, 2420

Shao, Z. Y., Xiao, Q. B., Shen, S. Y., et al. 2007, ApJ, 659, 1159

Stoughton, C., Lupton, R. H., Bernardi, M., et al. 2002, AJ, 123, 485

Thomas, D., Maraston, C., \& Bender, R. 2003, MNRAS, 339, 897

Trachternach, C., Bomans, D. J., Haberzettl, L., \& Dettmar, R. J. 2006, A\&A, 458,341

Tremonti, C. A., Heckman, T. M., Kauffmann, G., et al. 2004, ApJ, 613, 898

van den Hoek, L. B., de Blok, W. J. G., van der Hulst, J. M., \& de Jong, T. 2000, A\&A, 357, 397

Vorobyov, E. I., Shchekinov, Y., Bizyaev, D., Bomans, D., \& Dettmar, R. J. 2009, A\&A, 505, 483

Worthey, G., \& Ottaviani, D. L. 1997, ApJS, 111, 377

Zackrisson, E., Bergvall, N., \& Ostlin, G. 2005, A\&A, 435, 29

Zhong, G. H., Liang, Y. C., Liu, F. S., et al. 2008, MNRAS, 391, 986 